Milton Keynes University Hospital (MKUH) were included in this retrospective study. Patients included in this study underwent first time ERCP as indicated, including patients who developed PEP. PEP was defined according to consensus criteria. Patients who developed complications other than PEP, those who had failed procedure due to anatomical deformity, previous ERCP, or active pancreatitis at the time of ERCP were excluded.

Results A total of 260 patients who underwent ERCP between January 2016 and May 2017 were included in this retrospective study. After applying exclusion criteria 29 patients were excluded and 231 patients were included in the final analysis. PEP occurred in $8.6 \%(20 / 231)$ of all patients. Normal serum bilirubin was the only patient related risk factor which showed statistically significant association with PEP on univariate analysis $(\mathrm{P}=0.001)$. On univariate analysis of procedure risk factors we found that pancreatic guidewire cannulation, pancreatic contrast injection, and biliary metal stent insertion showed statistically significant association with PEP. Multivariate analysis after binary logistic regression analysis revealed that normal serum bilirubin $(\mathrm{P}<0.001)$ and bilary metal stent insertion $(\mathrm{P}=0.001)$ are independent risk factors for PEP. Pancreatic guidewire cannulation and pancreatic contrast injection increased the risk of PEP, but this was not statistically significant on the multivariate analysis.

Conclusions Normal serum bilirubin and biliary metal stent placement were independent risk factors for the occurrence of PEP. Further research is needed to investigate the etiological factors contributing to the development of PEP after biliary SEMS insertion. This will help to develop strategies to reduce SEMS related pancreatitis.

\section{PTH-002 OUT OF HOUR COLONOSCOPY; IS THE QUALITY AS GOOD AS IN HOUR COLONOSCOPY?}

Shimaa Afify*, Thomas Archer, Mo Thoufeeq. Sheffield Teaching Hospitals, UK

\subsection{6/gutjnl-2019-BSGAbstracts.27}

Introduction Colonoscopy is the 'gold standard' investigation for assessment of the large bowel which detects and prevents colorectal cancer, as well as non-neoplastic conditions. The quality of UK colonoscopy has improved as in 2013 The Joint Advisory Group (JAG) on GI Endoscopy suggests monitoring key performance indicators such as cecal intubation rate (CIR) and the adenoma detection rate (ADR). There has been a drive to improve utilization of endoscopy units nationwide by offering out of hours $(\mathrm{OOH})$ endoscopy to deal with increasing service demands. Our aim was to investigate the quality of colonoscopies carried out during evening and Saturday lists in our unit and compare against BSG standards of quality for colonoscopies. The endoscopy team consisted of our team.

Method We retrospectively collected and analyzed demographical and procedure related data for non- screening colonoscopies performed between January 2016 and April 2018. Procedures were grouped according to the day of the week and timing of session (weekday, evening and Saturday). We also compared those performed by advanced colonoscopist and non-advanced colonoscopist. Advanced colonoscopist was defined as a BCSP colonoscopist or an advanced EMR colonscopists.

Results There were a total of 13642 colonoscopies that were carried out. $26.3 \%(n=3601)$ involved patients were $>70$ years and $73.7 \%(n=10041)$ were $<70$ years. Table 1 summarizes the differences between weekdays, evening and Saturdays' colonoscopies regarding the CIR and ADR. We noted that the KPIs (ADR, CIR) met the JAG standards. Advanced colonoscopists had better KPIs when compared to the non-advanced colonoscopists.

Conclusion JAG standards were maintained during colonoscopies done on weekdays, evening and Saturdays. Advanced colonoscopists had higher CIR and ADRs.

\begin{tabular}{|c|c|c|c|c|c|c|c|c|}
\hline & & \multicolumn{2}{|c|}{ Weekdays } & \multicolumn{2}{|c|}{ Evenings } & \multicolumn{3}{|c|}{ Saturdays } \\
\hline & & No. & $\begin{array}{l}\text { Rate } \\
(\%)\end{array}$ & No. & $\begin{array}{l}\text { Rate } \\
(\%)\end{array}$ & No. & $\begin{array}{l}\text { Rate } \\
(\%)\end{array}$ & $\begin{array}{l}\mathrm{p} \\
\text { value }\end{array}$ \\
\hline \multirow[t]{2}{*}{ Total } & $A D R$ & 10775 & 28.5 & 1397 & 30.2 & 1469 & 24.6 & $0.002^{\mathrm{a}}$ \\
\hline & CIR & & 90.4 & & 90.6 & & 91.4 & 0.495 \\
\hline Advanced & $A D R$ & 954 & 42.6 & 136 & 32.4 & 197 & 34.0 & $0.012^{\mathrm{b}}$ \\
\hline colonoscopist & CIR & & 93.7 & & 96.3 & & 94.9 & 0.423 \\
\hline Non-advanced & ADR & 9821 & 27.1 & 1261 & 23.5 & 1272 & 23.1 & $0.001^{c}$ \\
\hline colonoscopist & CIR & & 90.1 & & 90.0 & & 90.8 & 0.704 \\
\hline advanced vs. & $p$ value (ADR) & $<0.001$ & & 0.022 & & 0.001 & & \\
\hline $\begin{array}{l}\text { Non-advanced } \\
\text { colonoscopist }\end{array}$ & $\mathrm{p}$ value $(\mathrm{CIR})$ & $<0.001$ & & 0.016 & & 0.055 & & \\
\hline
\end{tabular}

CIR: Caecal intubation rate, ADR: adenoma detection rate, a: Saturday is statistically significant than other days in ADR, b: all are statistically significant, c: week days are statistically significant from evenings and Saturdays

\section{PTH-003 DOES SIZE MATTER: HOW ACCURATE ARE OUR POLYP- SIZING METHODS?}

${ }^{1}$ Lulia Al-Hillawi ${ }^{*}$ 'Sun Mi Ha, ${ }^{2}$ Amir Saifuddin, ${ }^{2}$ Debra Uttridge, ${ }^{2}$ Chirag Kothari, ${ }^{1}$ Bijay Baburajan. 'Maidstone And Tunbridge Wells NHS Trust, Maidstone, UK; ${ }^{2}$ Dartford and Gravesham NHS Trust, Dartford, UK

\subsection{6/gutjnl-2019-BSGAbstracts.28}

Introduction Correct sizing of a colonic polyp at the time of lower gastrointestinal (LGI) endoscopy is key in dictating polypectomy method, use of tattoo, and future surveillance in colorectal cancer prevention (CRC). There are no accepted standards for how to size a polyp and therefore it is endoscopist-dependent and subject to inter-observer variation. Visual estimation (VE), or the use of open forceps (OF) to are the two most widely-used methods. There are limited studies on polyp-sizing at LGI endoscopy but they would suggest that the use of a measurement guide such as OF would improve size estimation. Our aim was to ascertain which polyp-sizing technique method is preferred. A secondary aim was to see if those using OF knew the size of the forceps being used.

Methods A 6 question survey was completed by endoscopists and nursing staff from Endoscopy Departments in 3 hospitals in West Kent using an online survey tool. Questions included asking for the respondent to identify how to position the scope and forceps based on photographs, and to identify the correct size of the OF when fully open.

The size of the open forceps of the Boston Scientific $(C)$ Standard $2.8 \mathrm{~mm}$ Radial $\mathrm{Jaw}^{\mathrm{TM}_{4}}$ single-use biopsy forceps was measured against a standard UK ruler.

Results There were a total of 27 respondents: $81 \%$ were endoscopists and 19\% nurse observers. 6 trainee endoscopists completed the survey. 
$67 \%$ used visual estimation to size polyps and 30\% used the open forceps technique. There was a discrepancy in how respondents would position the scope and forceps to size the polyp with no statistical significance. 22\% admitted they would not know which forceps were used in their department. Only $22 \%$ correctly identified the size of the OF (range $2 \mathrm{~mm}-8 \mathrm{~mm})$.

Of those endoscopists who perform >500 LGI endoscopies/year, 50\% used OF and 50\% VE. This group were more likely to correctly size OF. Trainee endoscopists performing only 50-100 LGI endoscopies mostly used VE which raises the question of whether they correctly size polyps given their limited experience.

Conclusion This small observational survey shows that the majority of endoscopists are likely to be incorrectly sizing polyps at LGI endoscopy. The majority of our respondents use VE which has been previously shown to be inferior to the OF technique. However, as demonstrated in this survey, our endoscopy staff do not know the correct size of the OF. Incorrect polyp sizing at LGI endoscopy could have far-reaching consequences on CRC prevention. Further work should be considered to develop a standardized technique for polyp sizing at the time of LGI endoscopy and to determine if training should focus more on this important skill.

\section{PTH-004 EFFICACY AND SAFETY OF ARGON PLASMA COAGULATION FOR THE TREATMENT OF RADIATION PROCTOPATHY}

Amjad Ali*, Muhammad Khan, Byron Theron, Andrew Davis, Alex Moran. Northern Devon Healthcare, Barnstaple, UK

\subsection{6/gutjnl-2019-BSGAbstracts.29}

Introduction Argon plasma coagulation (APC) is probably the most commonly used endoscopic treatment utilised for rectal bleeding due to radiation proctopathy (RP) but there are concerns about its efficacy and safety

Method Patients treated with APC for RP were identified from the endoscopy unit software database (Unisoft) over a 6 year period from 2011-2016. The case notes of each patient were obtained and the electronic patient record was also scrutinised in regard to symptoms, response to treatment and any complications.

Results 31 patients were identified that had APC for RP (median age 72, range 49-82) with all the 27 male patients having had radiotherapy for prostate cancer. There were four women that had APC for RP, 3 having had radiotherapy for cervical cancer and one having had radiotherapy for anal cancer. 28 of the 31 patients were referred for lower GI endoscopy primarily because of rectal bleeding. The number of APC treatments ranged from 1 to 7 (see figure 1) but only $10 / 31$ (32\%) required more than 3 sessions. Rectal bleeding was documented to have resolved in $28 / 31$. In 2 patients it was unclear if rectal bleeding had resolved but the endoscopy report stated for both patients that there were only minimal RP changes and there was no need for further APC. One patient did have some persistent minor rectal bleeding but this was not thought to relate to the residual RP and APC was not given. There was only one minor endoscopic complication with small rectal ulcers noted after APC but this was not symptomatic and resolved spontaneously by the time of repeat endoscopy. APC was provided at a flow rate of $0.6 \mathrm{~L} / \mathrm{min}$ and a power setting of $30 \mathrm{~W}$ for all patients.

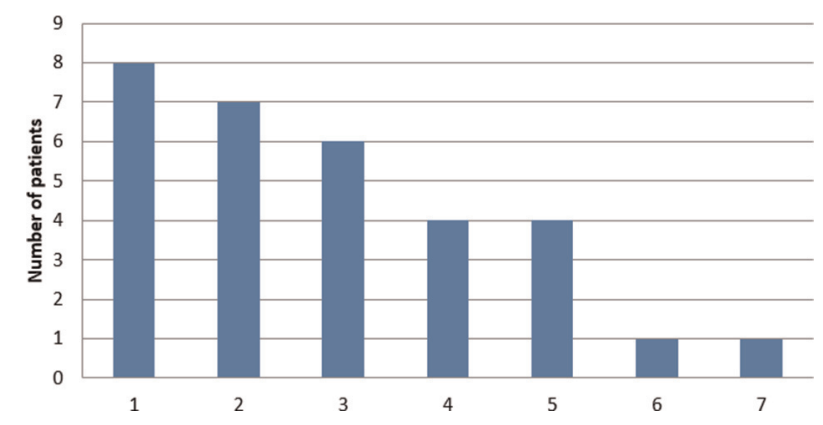

Abstract PTH-004 Figure 1 Number of APC treatments

Conclusions This long-term retrospective study has shown that only 10/31 (32\%) patients required more than 3 APC sessions for symptom resolution and that treatment was efficacious with symptom resolution documented in nearly all patients. There was only one minor complication of small ulcers in the rectum after APC but this was not symptomatic and resolved spontaneously. In summary, APC is an effective and safe treatment for RP but may need multiple sessions to provide symptom resolution. APC could be considered as an alternative to medical treatments such as Sucralfate enemas.

\section{PTH-005 RELIABILITY OF POST-COLONOSCOPY COLORECTAL CANCER ALGORITHMS}

${ }^{1}$ David Beaton*, ${ }^{2}$ Matthew Rutter, ${ }^{2}$ losif Beintaris. ${ }^{1}$ Newcastle Upon Tyne NHS, Newcastle Upon Tyne, UK; ${ }^{2}$ North Tees and Hartlepool NHS, North Tees, UK

\subsection{6/gutjnl-2019-BSGAbstracts.30}

Introduction Post-colonoscopy colorectal cancer (PCCRC) rate is an important metric of colonoscopy quality. The World Endoscopy Organisation (WEO) recently published a consensus statement advising review of PCCRC cases, to facilitate categorisation of PCCRCs into interval or non-interval cancers and determine their most plausible explanation. However, it is still unclear if the relevant algorithms are reproducible in real life practice, or how the respective plausible explanations are affected by factors such as reported adenoma location.

This study aims to test the inter-rater reliability for both categorisation of PCCRC and most plausible explanation of cause; it will also test whether altering the location of previously seen adenomas to neighbouring segments of the colon impacts on most plausible explanation categorisation.

Methods Colorectal cancer (CRC) cases diagnosed from January 2015 to December 2016 in a single trust were identified and each case was cross-referenced with online endoscopy and pathology databases to analyse cases of PCCRC. PCCRC definition was as per WEO consensus statement. Two assessors independently reviewed each PCCRC case, and agreement was measured using Cohen's kappa (k).

Results Among 527 patients with CRC, 35 cases of PCCRC were identified; mean age was 71 years (range 48-87) and 21 $(60 \%)$ of cases were males.

Categorisation of most plausible explanations is seen in table 1. Consistent most plausible explanation was found in $33 / 35$ cases, $\mathrm{k}=.903$ (95\% CI, .839 to .967), $\mathrm{p}<0.0005$. 